\title{
Norois
}

Environnement, aménagement, société

$221 \mid 2011$

Innovations et agricultures urbaines durables

\section{Nord et Sud face aux crises. De nouveaux réseaux maraîchers métropolitains au service d'une agriculture de proximité : les cas de Buenos Aires et Stockholm}

North and South facing crisis. New metropolitan market gardening networks in the service of proximity farming. The cases of Buenos Aires and Stockholm

Camille Hochedez et Julie Le Gall

\section{OpenEdition Journals}

\author{
Édition électronique \\ URL : http://journals.openedition.org/norois/3766 \\ DOI : $10.4000 /$ norois.3766 \\ ISBN : 978-2-7535-1838-4 \\ ISSN : 1760-8546 \\ Éditeur \\ Presses universitaires de Rennes \\ Édition imprimée \\ Date de publication : 30 décembre 2011 \\ Pagination : 25-38 \\ ISBN : 978-2-7535-1786-8 \\ ISSN : 0029-182X \\ Référence électronique \\ Camille Hochedez et Julie Le Gall, « Nord et Sud face aux crises. De nouveaux réseaux maraîchers \\ métropolitains au service d'une agriculture de proximité : les cas de Buenos Aires et Stockholm », \\ Norois [En ligne], 221 | 2011, mis en ligne le 30 décembre 2013, consulté le 30 avril 2019. URL : http:// \\ journals.openedition.org/norois/3766 ; DOI : 10.4000/norois.3766
}




\title{
NORd ET SUd FACE AUX CRISES
}

\section{DE NOUVEAUX RÉSEAUX MARAîCHERS MÉTROPOLITAINS \\ AU SERVICE D'UNE AGRICULTURE DE PROXIMITÉ : les cas de Buenos Aires et Stockholm}

\author{
Camille Hochedez \\ BioGéophile - UMR 5600 Environnement, Ville, Société \\ (Université de Lyon - ENS de Lyon, site Descartes), \\ 15, parvis René-Descartes, BP 7000 - 69342 Lyon cedex 07, France \\ camille.hochedez@ens-lyon.fr \\ Julie Le Gall \\ UMR 8586 PRODIG \\ (Université Paris 1 Panthéon-Sorbonne), \\ 12, place du Panthéon - 75005 Paris \\ julielegall@gmail.com
}

\section{RÉSUMÉ}

L'Argentine comme la Suède ont été touchées plus ou moins récemment par diverses crises multiformes qui ont entraîné l'évolution de leur appareil productif, notamment agricole. Ces moments de rupture et de perturbation intenses sont porteurs de recompositions territoriales importantes à l'échelle de leurs métropoles, dans l'Aire métropolitaine de Buenos Aires et dans la région de Stockholm. Par l'étude des mutations de leurs réseaux d'approvisionnement en produits frais (légumes, biologiques et de qualité à Stockholm, conventionnels à Buenos Aires), ce travail propose un éclairage Nord/Sud des réponses à la crise des systèmes agricoles. Dans ces deux métropoles, l'innovation provient d'acteurs extérieurs au système agricole où ils s'installent (Boliviens à Buenos Aires, néo-ruraux à Stockholm), et organisés en réseaux (communautaires et associatifs). Ils mettent en place des agricultures pour la ville plus intensives et plus familiales, et s'appuient sur des nouvelles structures de commercialisation en réseau, plus directes (marchés de gros à Buenos Aires, marchés urbains de producteurs à Stockholm). Issues d'en bas, ces innovations agricoles et sociales sont reprises dans un second temps par l'État et les institutions politiques, disposant d'outils législatifs pour entériner les recompositions territoriales face aux enjeux fonciers. Ces attitudes politiques récentes répondent à un même besoin de durabilité qui est autant socio-territoriale qu'environnementale.

MOTS CLÉ : réseaux - innovation-gouvernance-maraîchage-Buenos Aires - Stockholm

\section{ABSTRACT}

North and South facing crisis. New metropolitan market gardening networks in the service of proximity farming. The cases of Buenos Aires and Stockholm

Several economical, political and social crises affected both Argentina and Sweden more or less recently. Those times of intense disturbances implied several changes in their pro- 
duction system, notably in their farming system. These break points are the bearer of some important changes of metropolitan territories pattern on a regional scale in the metropolitan regions of Buenos Aires and Stockholm. We propose a study of two ways to react to the farming system crisis in Northern or in Southern countries in focusing on changing supplying networks of fresh products (organic and quality products in Stockholm and conventional ones in Buenos Aires). In both cases, agricultural systems are changed by actors organized into community networks, coming from the outside of agricultural systems where they settle in. They introduce a smaller scale, familial and intensive agriculture and work to create shorter supplying thus directly connecting production and commercialization (wholesale market in Buenos Aires, farmers' markets in Stockholm). These bottom-up agricultural and social innovations are taken up by the State and the political authorities, which use legislative tools to ratify territorial resettings vis-a-vis land stakes. Both political attitudes are meeting the same need for socio-territorial sustainability, as much as environmental sustainability.

KEYWORDS : networks - innovation - governance - market gardening - Buenos Aires - Stockholm

L'agriculture urbaine et périurbaine revient au cœur des débats alors que pour la première fois en 2008, la part de la population urbaine mondiale a dépassé celle de la population rurale (FAO, 2009). Même s'il n'existe pas encore de définition universellement acceptée, la FAO appelle « agriculture urbaine et périurbaine » «les pratiques agricoles dans les villes et autour des villes qui utilisent des ressources (terre, eau, énergie, main-d'œuvre) pouvant également servir à d'autres usages pour satisfaire les besoins de la population urbaine » (FAO, 2009). Suite aux crises alimentaires de grande ampleur (notamment celles de 2007-2008), les nouvelles problématiques des réflexions scientifiques engagées insistent sur la nécessité d’innover dans le domaine alimentaire et de limiter à long terme les inégalités sociales d'accès à l'alimentation. Cela passe notamment par le développement d'une agriculture de proximité, au Nord à cause de l'élévation des cours du pétrole, de la disparition du tissu social agricole qui rend ces pays de plus en plus dépendants d'autres espaces de production dans le monde; au Sud, parce que les crises alimentaires touchent en priorité les populations pauvres urbaines des grandes villes qui, par leur nombre et leur capacité de réaction, peuvent déstabiliser un Etat (Houtard, 2009). Dans un contexte post-crise, l'agriculture tient un rôle qui va au-delà de la production des aliments : elle contribue à l'amélioration des régimes alimentaires, au maintien des emplois et des revenus, tout en participant à une paix sociale (Bricas et Daviron, 2008).

Or l'Argentine et la Suède sont restées à l'écart des crises alimentaires récentes : leur situation alimentaire n'est pas inquiétante, ni structurellement, ni conjoncturellement. Pourtant, elles ont été touchées plus ou moins récemment par d'autres crises, multiples et multiformes (économiques, politiques, sociales), qui ont entraîné l'évolution de leur appareil productif, notamment agricole. Ces moments de rupture et de perturbation intenses sont porteurs de recompositions territoriales à l'échelle régionale de leurs métropoles, dans la Région métropolitaine de Buenos Aires et dans la région urbaine de Stockholm. Ils ouvrent une réflexion sur l'alimentation de ces populations urbaines et leur approvisionnement en produits frais. Les précédentes sorties de crise vécues par ces pays pourraient dès lors servir d'exemples pour éclairer les risques alimentaires à venir dans d'autres pays.

Les analyses s'accordent aussi sur la nécessité de ne pas considérer la seule production agricole pour expliquer ou prévenir les crises : il est important d'observer également le devenir des productions. La notion d'approvisionnement est définie par D. Douzant-Rosenfeld et P. Grandjean (1996) comme « à la fois le processus d'acheminement des productions jusqu'à la ville et leur 
distribution à l'intérieur de l'espace urbain. » Il s'agit d'une notion « carrefour », prenant en compte à la fois les dimensions économiques (la production, la circulation, les prix), sociales (accès inégal à l'alimentation), culturelles (habitudes de consommation) et spatiales (répartition des revenus, lieux de commercialisation, flux). Ainsi, la thématique de l'agriculture périurbaine n'est pas nouvelle mais les enjeux ont évolué et, plus que jamais, l'étude de l'approvisionnement lui apparaît complémentaire. Conformément à ces angles d'analyse, ce travail propose un éclairage Nord/Sud des réponses à la crise des systèmes agricoles à travers l'étude des mutations des réseaux d'approvisionnement des capitales suédoise et argentine en produits frais (légumes, biologiques et de qualité à Stockholm, conventionnels à Buenos Aires) et des relations qui existent entre lieux de production, de commercialisation et de consommation.

Les terrains sont trop différents pour que la comparaison des agricultures périurbaines ou des types d'espaces maraîchers soit fructueuse. En revanche, les logiques spatiales affectant les deux espaces d'étude et les réponses aux pressions métropolitaines présentent des similitudes : cet éclairage Nord/Sud permet de réfléchir aux mécanismes favorisant le maintien des espaces maraîchers pour nourrir les grandes métropoles, aux acteurs impliqués et au rôle des politiques dans ce maintien. Au-delà de l'étude de cas, deux questions émergent : comment le maintien d'une agriculture pour la ville garantit à la fois un meilleur accès à l'alimentation pour tous (en quantité et en qualité) et un équilibre entre les différents usages (productifs, immobiliers, récréatifs) des espaces périphériques métropolitains? Comment les dynamiques spatiales liées à l'innovation dans les systèmes maraîchers construisent des territoires métropolitains plus durables?

Nous apporterons des éléments de réponse en utilisant les résultats tirés d'entretiens menés auprès d'un échantillon d'agriculteurs (27 pour le cas argentin, 45 pour le cas suédois) appartenant à des réseaux (circuits courts dans le cas de Stockholm, circuits boliviens dans le cas de Buenos Aires), auprès de leurs responsables, des organisations professionnelles agricoles, des collectivités locales et des organismes de recherche agricoles et agronomiques. Ces résultats ont ensuite été croisés avec la consultation de documents d'aménagement locaux. Le travail a été mené dans le cadre de deux thèses de doctorat.

\section{Des crises multiples et multiformes}

Les systèmes agricoles argentin et suédois ont été touchés par de nombreuses crises qui ont entraîné leur redéfinition tout en assurant leur maintien.

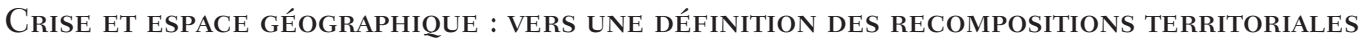

La crise désigne en général un moment critique et implique nécessairement le changement et la transformation du système ${ }^{1}$. Les économistes l'ayant théorisé, tels que Kondratieff et Schumpeter, insistent sur le fait que l'innovation est le moment qui permet de passer d'une phase B (descendante) à une phase A (ascendante), donc de sortir provisoirement de la crise. Crise et innovation sont deux notions qui, en géographie, sont affiliées à celles de résilience et de développement durable. Ces deux dernières permettent d'envisager le maintien d'un système dans le temps long, et de penser les permanences et les changements d'un système géographique. La résilience est définie comme la capacité d'un système à pouvoir intégrer dans son fonctionnement une perturbation, sans pour autant changer de structure qualitative (Aschan-Leygonie, 2000). Un système est dit résilient s'il adapte sa structure au changement tout en conservant la même trajectoire (Holling, 1973). Appliquée à la géographie, la notion de résilience peut s'envisager à travers celle de recomposition territoriale, entendue comme les dynamiques spatiales issues d'en bas (de "l'intérieur ») ou d'en haut (pouvoirs territoriaux) en réponse à des chocs brefs ou des processus longs, brutaux

1. Dans le dictionnaire de géographie de R. Brunet et al. (1992) (seul dictionnaire de géographie à avoir une entrée «crise »), la crise est définie comme un «moment d'extrême tension, de paroxysme, de conflit, de changement » (p. 125) 
dans les deux cas, qui modifient profondément et durablement l'interaction entre un espace et sa société, et, par conséquent, l'organisation de cet espace (Le Gall et al., 2009).

\section{À Buenos Aires, trois décenNies d’insertion deS MigRants boliviens}

Comme les autres grandes villes des pays du Sud, Buenos Aires a vu subsister sa ceinture verte périphérique, espace chargé d'alimenter la capitale en légumes frais. La capitale argentine présente une forte spécificité : l'activité maraîchère familiale est entre les mains des migrants boliviens et de leurs descendants (fig. 1). En trente ans, les voisins du Cône sud sont devenus les acteurs incontournables de l'alimentation en légumes de Buenos Aires, une activité qui avait toujours été entre les mains des Italiens, des Japonais, des Portugais. Ils occupent à ce jour l'ensemble des espaces maraîchers de production, de commercialisation de gros et de vente au détail, représentant 42 \% des producteurs maraîchers de la région métropolitaine (et dominant de façon hégémonique la production familiale) (Benencia, 2009, Ministerio De Asuntos Ararios, Ministerio De Economia

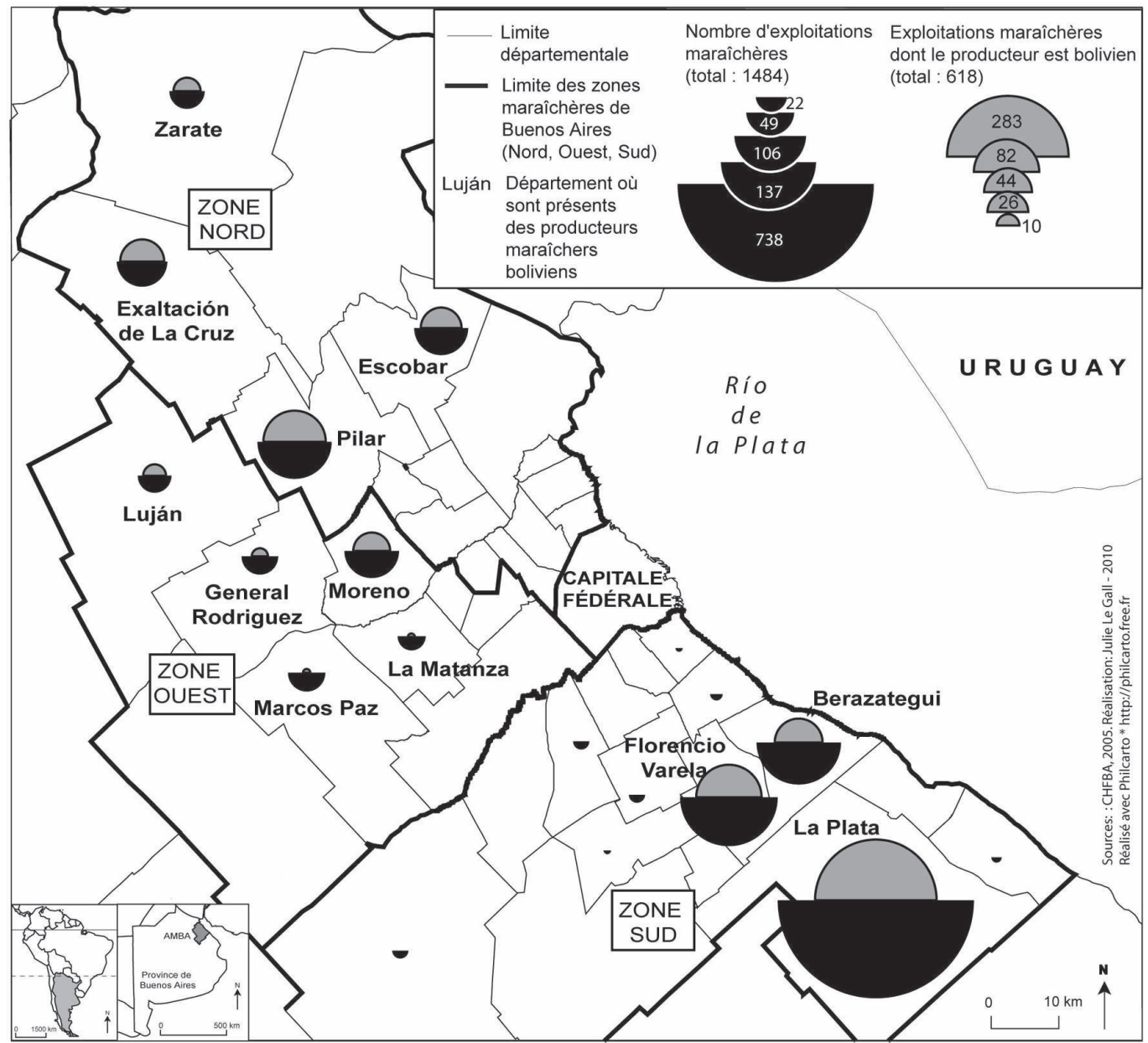

Figure 1 : Carte de localisation des espaces productifs de Buenos Aires Location map of the green belt of Buenos Aires 
et Gobierno De La Provincia De Buenos Aires, 2006, Pizarro, 2009) et entre 75 et $90 \%$ des commerçants grossistes de nombreux marchés de gros spécialisés en légumes (Le Gall, 2007).

Les Boliviens se sont insérés en profitant de trois retournements majeurs touchant directement ou indirectement les espaces maraîchers de production et de commercialisation :

- dans les années 1980, les crises internes au secteur maraîcher (surproduction et vieillissement des producteurs et commerçants sans reprise par leurs enfants) ont rendu nécessaire le recours à de nouveaux arrivants et permis leur ascension;

- dans les années 1990, les bouleversements profonds liés aux processus de mondialisation et métropolisation et à la néolibéralisation de l'économie et des politiques (dollarisation, facilité d'accès aux intrants et aux serres, amélioration des accès aux périphéries de la capitale, ouverture du Mercosur, entre autres mutations) ont non seulement attiré les Boliviens en Argentine mais leur ont aussi permis d'accumuler rapidement un capital économique, de développer une agriculture maraîchère et des stratégies commerciales compétitives et de circuler plus facilement au sein de la région entre zones de production et lieux de commercialisation;

- lors de la crise multiforme de 2001, qui a déboussolé l'ensemble du pays, les Boliviens de l'activité maraîchère, insérés dans des circuits économiques informels, ont plutôt mieux résisté que d'autres et ont bénéficié de nouveaux abandons d'exploitations et de commerces, avant de profiter de la reprise à partir de 2003.

À chaque étape une niche s'est donc libérée dans les espaces de production ou de commercialisation qui a pu être investie et recomposée par les Boliviens et leurs descendants.

L'ensemble des transformations socio-spatiales de la métropole et du secteur maraîcher entraîne une restructuration profonde des espaces de production et de commercialisation. Avec la croissance démographique, l'avancée du front d'urbanisation et le déploiement des réseaux routiers, on assiste à un redéploiement des espaces de production vers la quatrième couronne qui s'accompagne, sous l'effet des pressions métropolitaines, d'une concentration en deux « îles productives », la zone Nord autour des départements de Pilar et Escobar, et la zone Sud à La Plata, rompant ainsi avec le modèle de ceinture verte qui prédominait jusqu'alors (fig. 1). La zone Sud se spécialise dans la production sous serre, tandis que la zone Nord demeure une zone de cultures de plein champ, en restructuration. Dans les espaces de commercialisation, les structures de vente des légumes ne suivent pas le processus de concentration des commerces alimentaires mais se multiplient (nouveaux marchés de gros, supermarchés, magasins de primeurs, vente de rue), sans doute du fait de l'hétérogénéité de la demande : les disparités des niveaux de revenus exigent la présence de types de commerces variés et plus ou moins formels. De même, pour faire face à l'augmentation des baux locatifs, les commerçants partagent leurs emplacements ou leurs locaux, provoquant une densification des espaces maraîchers commerciaux.

\section{À STOCKHOLM, LE DÉVELOPPEMENT D'UNE AGRICULTURE BIOLOGIQUE FAMILIALE}

En Suède, les agriculteurs biologiques approvisionnant la capitale en produits frais ont peu à peu gagné du terrain suite à une série de transformations du système agricole depuis le milieu du XIX ${ }^{\mathrm{e}}$ siècle.

- Des années 1880 aux années 1950 : crise de sous-production du système agricole traditionnel liée à la surpopulation, puis crise de surproduction des années 1930 liée à la concurrence de l'agriculture des pays du Nouveau Monde. Ces crises ont entraîné à partir des années 1950 la réorganisation et la rationalisation de la production avec l'apparition de grandes coopératives de producteurs, et finalement la mise en place d'un modèle agricole industriel moderne, surtout en Suède méridionale et dans une moindre mesure en Suède centrale.

- À partir des années 1980 : crise écologique liée à l'agriculture intensive. Une réforme de la politique agricole suédoise a été entamée en 1990 pour répondre à cette crise : dérégulation interne, forte diminution du soutien aux prix et introduction de subventions pour les biens publics (développement régional, paysage). Cette crise écologique a été renforcée dans les années 1990 
par une série de crises alimentaires mondiales (vache folle, grippe aviaire, OGM) qui ont rendu les consommateurs de plus en plus méfiants par rapport au système alimentaire mondial, et de plus en plus demandeurs en traçabilité et en produits locaux et de qualité.

- Crise du modèle agricole européen dans les années 1990, subie par la Suède en 1995 lors de son adhésion à l'Union Européenne. La crise européenne agricole est une crise de surproduction, entraînant de nombreuses réformes de la PAC, et la redéfinition du modèle agricole européen vers une agriculture de qualité. Le tournant a été pris en 2003, avec d'une part le passage au paiement unique à l'exploitation, et d'autre part l'introduction du principe d'écoconditionnalité dans le paiement des aides (Jordbruksdepartementet, 2006).

Les évolutions du système productif agricole ont favorisé le développement de l'agriculture biologique, en partie grâce aux subventions, aux politiques et aux règlements (Règlement nº 2092/91 de l'Union européenne) mis en place à l'échelle européenne. Ainsi, en Suède, les surfaces cultivées en mode de production biologique sont passées de 50000 ha en 1995 à 215970 ha en 2007 (Eurostat). L'agriculture biologique est notamment plus développée dans les départements de la Suède centrale, avec une forte présence dans les départements voisins de celui de Stockholm (fig. 2).

Paradoxalement, les circuits courts de commercialisation des produits biologiques sont encore peu importants. En effet, les grandes surfaces dominent le marché des produits alimentaires biologiques, qui ne représente que $2 \%$ du marché alimentaire suédois en 2007; seules $7 \%$ de ces ventes se font directement du producteur au consommateur (Jordbruksverket, 2009). Mais des filières courtes d'approvisionnement en produits maraîchers se structurent depuis les années

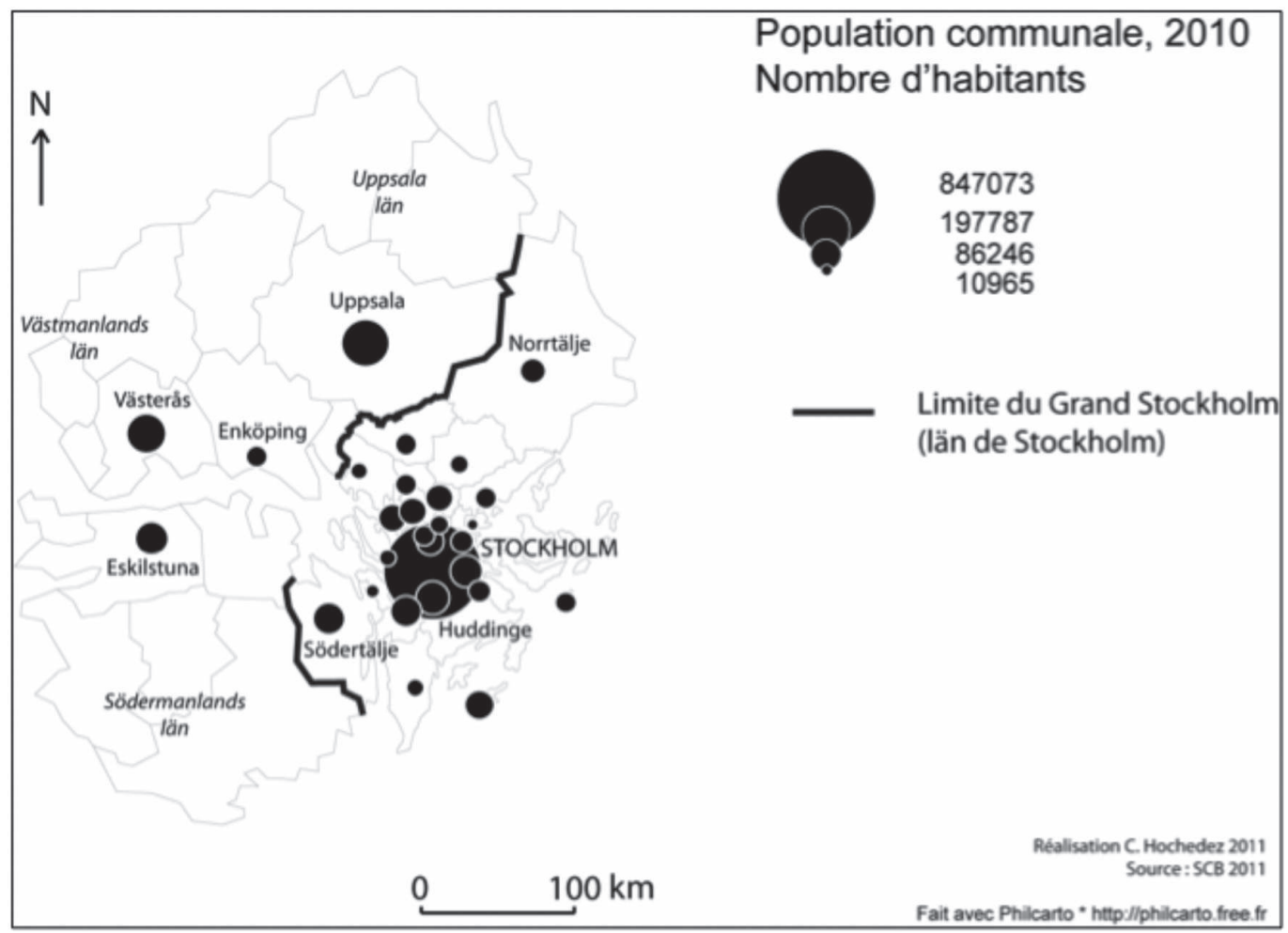

Figure 2 : Carte de localisation de la région de Stockholm Location map of the Stockholm area 
2000 dans et autour de la capitale. Plusieurs exemples ont été étudiés : Roslaglådan et Ekolådan (deux systèmes de paniers de fruits et légumes biologiques au nord et au sud de la capitale), et les marchés fermiers Bondens Egen Marknad et Street, tous deux localisés à Stockholm et proposant la vente par les fermiers eux-mêmes de produits locaux majoritairement biologiques, au printemps et à l'automne.

À Buenos Aires comme à Stockholm, les crises mêlent donc facteurs internes et externes à l'activité maraîchère et proviennent d'un jeu d'échelles complexe entre les espaces maraîchers, les régions métropolitaines, les Etats et les préoccupations et processus mondiaux. Elles opèrent dans des temporalités différentes : retournements de situation brutaux et reprises rapides sur le temps court des trente dernières années en Argentine, transformations lentes et en profondeur depuis le XIX ${ }^{\mathrm{e}}$ siècle en Suède. Les crises agricoles ont eu pour conséquence de complexifier les systèmes agricoles et alimentaires nationaux, et de mettre sur le devant de la scène une petite agriculture à proximité de la métropole, biologique ou non, porteuse d'innovations.

\section{Nouveaux agriculteurs, nouvelles agricultures}

Au-delà de son rôle nourricier, l'agriculture de proximité joue également un rôle de pourvoyeuse de services pour la société. Produisant de nouvelles fonctions pour la ville, notamment en termes de qualité de vie (Poulot, 2008), cette activité est alors perçue comme un rempart face à l'urbanisation, et participerait à la construction de la ville durable (Fleury et Donadieu, 1997). Durabilité et gouvernance territoriale sont aujourd'hui les deux angles d'étude principaux de l'agriculture de proximité (Pierre et al., 2008). Or ces deux dimensions sont inégalement présentes dans nos études de cas. Dans le prolongement des questionnements sur la gouvernance à l'œuvre dans l'agriculture de proximité, l'étude des acteurs et des pratiques agricoles à Buenos Aires et à Stockholm montre comment ils font évoluer les systèmes agricoles de l'intérieur.

\section{DES ACTEURS EXTERIEURS AU SYSTÈME AGRICOLE OÙ ILS S'INSTALLENT}

Autour des deux capitales se sont installés de «nouveaux » agriculteurs dans les périphéries maraîchères, étrangers par leur nationalité en Argentine, étrangers au monde agricole en Suède.

Les Boliviens, originaires des montagnes, s'extasient devant les facilités des pratiques agricoles de la Pampa, plane et irriguée. Le type d'agriculture qu'ils exercent et l'arrivée dans un pays où l'on ne parle pas, contrairement aux pays andins, de «campesinos » («paysans ») les détachent de ce statut qui leur était traditionnellement octroyé en Bolivie. On parle même d'eux à Buenos Aires comme des «farmers ", si l'on souhaite souligner le caractère très intensif de leurs pratiques. Le statut de producteur ne va néanmoins pas de soi : les Boliviens ont gravi une échelle sociale en étant d'abord ouvriers agricoles puis métayers et quand ils passent producteurs, ils restent locataires car l'accès au foncier est rare et compliqué.

À Stockholm, les producteurs sur les marchés de produits biologiques sont souvent des urbains ou des néo-ruraux, n’ayant pas de racines agricoles dans leur famille (la moitié des agriculteurs biologiques interrogés n’ont aucune ascendance familiale agricole). $83 \%$ des agriculteurs interrogés dans le cadre des marchés ont exercé un ou plusieurs emplois avant de s’installer en tant qu'agriculteurs, contribuant à renforcer davantage leur ancrage dans le monde urbain. Les autres (17\%) se sont installés directement en tant qu'agriculteurs.

Ainsi, on peut qualifier les producteurs biologiques de Stockholm de « néophytes dans l'agriculture " tandis que les Boliviens maraîchers de Buenos Aires sont «néophytes dans un certain type d'agriculture ». Les trajectoires personnelles étudiées indiquent le rôle du facteur migratoire, de la ville à la campagne en Suède, et de la Bolivie à l'Argentine, pour déclencher un mouvement entrepreneurial. Ces nouvelles populations installées en milieu rural périurbain apportent avec elles des pratiques (urbaines et écologiques dans un cas, boliviennes dans l'autre) qui contribuent à les différencier du tissu agricole où elles s’implantent. Dans les deux cas, cette arrivée récente 
depuis un milieu et une activité autres suffit à insuffler une dynamique innovante aux espaces maraîchers périurbains investis.

\section{Des AGRicultures Plus intensiVEs, ET FAMiLiales}

Les nouveaux circuits d'approvisionnement s'appuient sur une forme d'agriculture différente du système dominant (national et régional).

La taille des exploitations constitue une première rupture dans les systèmes d'approvisionnement considérés. À Buenos Aires, si, dans les zones Nord et Sud, la superficie moyenne de l'ensemble des exploitations maraîchères avoisine les 5,5 ha (et plutôt les 20 ha pour les producteurs maraîchers traditionnels italiens et portugais rencontrés), les exploitations tenues par les Boliviens interrogés présentent une superficie deux fois moindre (au Nord) à cinq fois moindre (au Sud). À Stockholm, où existe une agriculture de loisir (phénomène des moonlight farmers), les exploitations des producteurs interrogés sont parfois à la limite de la définition statistique de l'agriculture (au moins $2 \mathrm{ha}$ ), si on se réfère à la médiane, soit moins de 1,4 ha ${ }^{2}$, alors que la moyenne des « exploitations » enquêtées (34,9 ha) renvoie plutôt à la taille moyenne d'une exploitation suédoise.

Ces exploitations sont également caractérisées par leur tendance à l'intensification. À Buenos Aires, les exploitations enquêtées sont à la fois plus productives et plus demandeuses en maind'œuvre du fait de la généralisation des cultures sous serre (Kebat, 2008). À Stockholm, la nouvelle agriculture commercialisant sur les circuits courts est peu productive mais assez intensive en main-d'œuvre. Les exploitations suédoises enquêtées ont recours en moyenne à deux personnes, chef d'exploitation inclus, ainsi qu'à au moins un saisonnier l'été. Le système de production biologique implique par contre une productivité et des rendements inférieurs du fait du non-recours aux engrais chimiques et de la rotation des cultures. La réintroduction de la polyculture, et de l'association cultures/élevage est aussi spécifique à ce système.

Cette intensification s'appuie sur une main-d'œuvre familiale. À Buenos Aires, l’implication de tous les membres de la famille, de l'enfant de deux ans au chef d'exploitation, fait la spécificité de la nouvelle activité maraîchère productive. Les espaces de commercialisation sont quant à eux marqués par leur féminisation. Ce système familial et les économies qu'il entraîne pourraient expliquer la capacité des Boliviens à mobiliser rapidement un capital et à grimper les échelons sociaux. Actuellement, il constitue aussi une réponse au manque cruel d'ouvriers et de saisonniers agricoles $^{3}$. Dans le cas suédois, l'agriculture est familiale et pluriactive. Les chefs d'exploitation reçoivent l'aide du conjoint voire des enfants (non rémunérés) pour les tâches de production, et plus souvent de commercialisation. L'agriculture est rarement la source principale de revenus du ménage et sa pratique à temps partiel est répandue en complément d'un autre emploi.

L'implication de nouveaux acteurs qui portent un regard neuf sur une activité de longue date constitue l'innovation qui permet de sortir des différentes crises. Elle a un effet d'entraînement sur l'ensemble du système d'approvisionnement.

\section{Des réseaux pour innover}

À Buenos Aires et à Stockholm, la diffusion de l'innovation marâichère prend la forme du réseau. Nous entendons par « réseau » l'ensemble des liens matériels, immatériels, sociaux, s'exprimant sur un espace donné par des échanges multilatéraux et donnant lieu à la construction de nœuds,

2. Cette moyenne, a priori élevée, tient au fait que plusieurs types d'exploitations (maraîchères, laitières, autre élevage) sont présents dans les réseaux de commercialisation étudiés. Ainsi, les plus grandes exploitations (correspondant à une activité d'élevage) ont une superficie de 140/150 ha, ce qui tire la moyenne des exploitations des réseaux vers le haut.

3. Du fait des améliorations du niveau de développement en Bolivie sous la présidence d’Evo Morales, et des difficultés économiques de l'Argentine depuis 2001, il est moins rentable pour les Boliviens de travailler ou de s'installer à Buenos Aires. Ceux qui continuent à le faire choisissent les secteurs du textile ou de la construction, moins éprouvants physiquement que l'agriculture. 
de lignes, de jeux d'acteurs à différentes échelles. Les réseaux sociaux ont facilité la connexion des espaces de production aux réseaux de commercialisation.

\section{DE NOUVELLES STRUCTURES POUR UNE CONNEXION PRODUCTION/COMMERCIALISATION}

Outre par son système de production, c'est aussi par les modes de commercialisation en réseau que l'agriculture périurbaine étudiée est innovante.

En Argentine, les Boliviens ont créé leurs propres marchés de gros (photo 1) à partir de la moitié des années 1990, au cœur des espaces de production (zone Nord). Ils ont aussi investi les anciens marchés de gros, en divisant les emplacements laissés vacants et en en créant de nouveaux dans les espaces vides. En ville, d'autres Boliviens spécialisés dans la commercialisation de détail créent leurs magasins de primeurs ou vendent à même la rue. À l'inverse, la forme du marché constitue une innovation en Suède en général et à Stockholm en particulier, car il n'existe plus qu'un seul marché quotidien de produits frais à Stockholm même (Hötorget). Ces nouveaux canaux de commercialisation sont adaptés au petit volume de production et surtout à la nécessité de tirer une forte rémunération lors de la vente, en supprimant les intermédiaires.

Ces marchés replacent l'agriculteur-paysan au cœur de l'acte commercial, puisqu'il est lui-même le vendeur. À Stockholm, les marchés de vente directe s'appuient sur des règles fermes : le vendeur est obligatoirement le producteur de ce qu'il vend (photo 2). À Buenos Aires, aucune règle n'est fixée mais les Boliviens grossistes en légumes verts sont soit des producteurs, soit des revendeurs s'approvisionnant directement auprès de ces mêmes producteurs. En outre, on voit aussi des

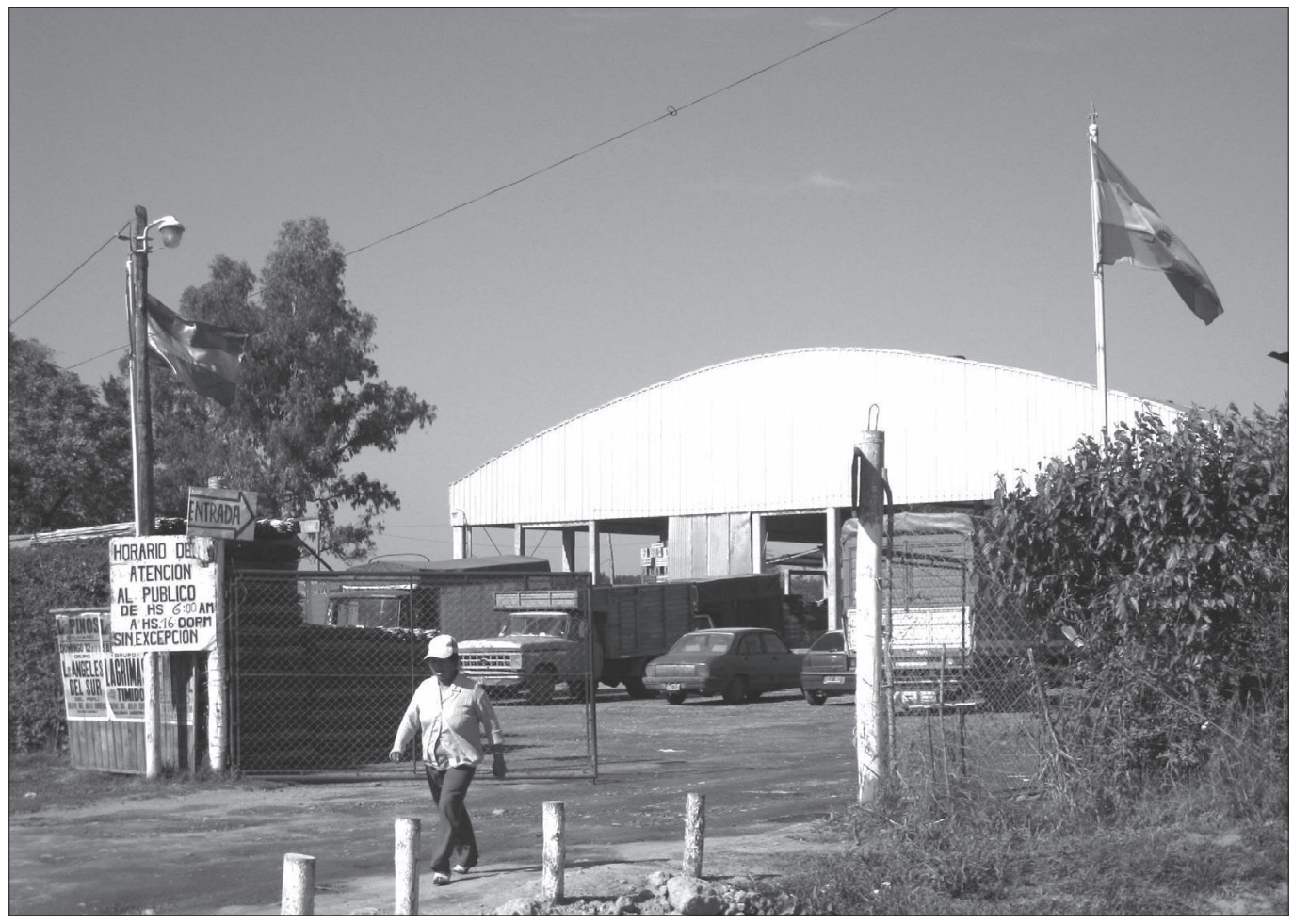

Photo 1 : Le marché de gros de Pilar, un nouveau marché crée par les Boliviens (cl. J. Le Gall - 2008)

The Bolivian wholesale market of Pilar 
particuliers vivant à proximité faire leur marché au marché de gros : on peut parler ainsi d'une « détaillisation » des marchés de gros. Dans les deux cas, les marchés sont des lieux privilégiés du contact entre paysans-commerçants et citadins (Chaléard, 2005). Ils sont l'occasion de parler de son produit, de ses méthodes de production, de son exploitation, aux citadins. La traçabilité est totale et satisfait les nouvelles demandes des consommateurs au Nord comme au Sud. De plus, à Stockholm se mêlent nature idéalisée, qui sent bon les traditions et le passé rustique, et valeurs écologiques autour du développement durable (photo 2). Les marchés fermiers utilisent l'idylle rurale pour ajouter de la valeur symbolique aux produits vendus (Guiu, 2007) et pour revaloriser le métier d'agriculture aux yeux des citadins des quartiers riches et gentrifiés (Östermalm et Södermalm). À Buenos Aires, les Boliviens sont valorisés pour la fraîcheur de leurs produits, ce qui marque une première reconnaissance du rôle de ces acteurs autrefois discriminés.

\section{RÉSEAUX ASSOCIATIFS, RÉSEAUX COMMUNAUTAIRES}

Dans les deux exemples, les réseaux de l'innovation s'appuient donc sur des réseaux sociaux déjà existants, selon les liens communautaires à Buenos Aires, selon les relations de proximité et professionnelles à Stockholm.

À Buenos Aires sont mis en évidence deux types de réseaux : les familles élargies et les associations à l'origine des marchés de gros, apparentées à des coopératives, mais qui sont aussi des lieux de rassemblement de la collectivité (fêtes, tournois sportifs). Les réseaux sociaux ont facilité la connexion des espaces de production aux réseaux de commercialisation et les circulations croissantes des Boliviens au sein de l'Aire métropolitaine. En effet, l'obtention d'un emplacement dans un marché de gros se fait généralement sur information d'un parent proche ou lointain, et

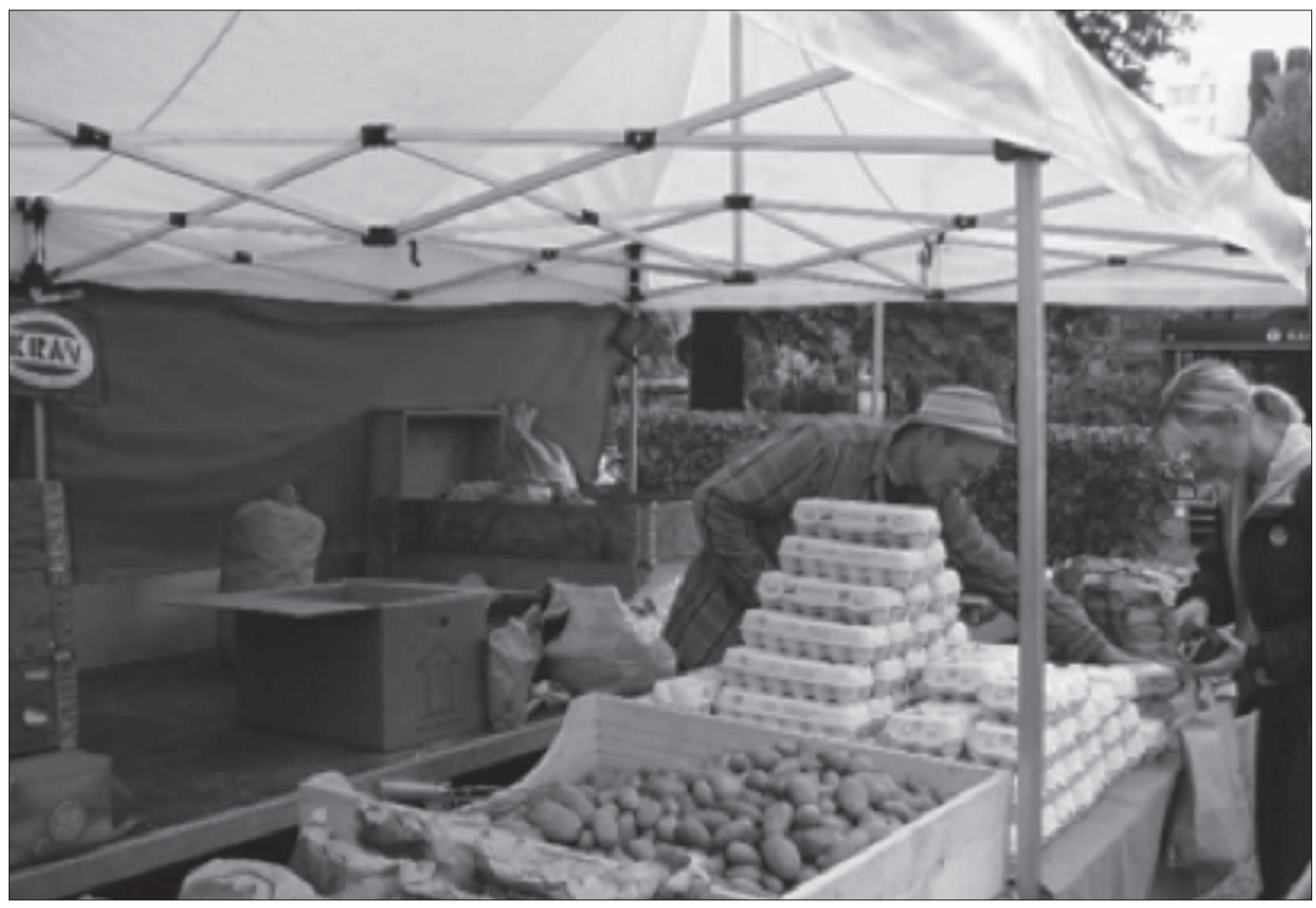

Photo 2 : Les marchés fermiers « Bondens Egen Marknad» à Stockholm (cl. C. Hochedez - 2008) Farmers' market «Bondens Egen Marknad » in Stockholm City 
la clientèle s'établit plus facilement entre Boliviens, qu’ils soient producteurs, détaillants et grossistes, parfois de façon spécifique entre Boliviens d'un même département d’origine.

En Suède, les réseaux regroupent producteurs, transformateurs, restaurateurs et magasins. Les agriculteurs viennent d'horizons géographiques différents, ce qui leur permet de s'insérer sur le marché, et d'écouler plus facilement leur production grâce aux contacts des différents agriculteurs du réseau. Ces structures associatives proposent des activités à leurs membres (formation, voyages d'étude) qui renforcent la sociabilité interne. De plus, elles s'étoffent par l'intégration de nouveaux membres : des voisins ou des connaissances personnelles (Hochedez, 2008).

Les nœuds de ces réseaux sociaux sont dans les deux cas des leaders, pourvus d'une âme d'entrepreneur, et capables de saisir les opportunités pour investir les niches économiques. Ils transforment leur espace de référence : le marché, la zone de production, deviennent des nœuds des réseaux d'approvisionnement. Surtout, ils peuvent porter sur la scène publique les innovations de leur groupe d'appartenance.

\section{Des attitudes politiques locales différenciées mais répondant à un même besoin de durabilité}

Les réseaux étudiés, porteurs d'innovations agricoles et sociales, sont le fruit d'initiatives issues d'en bas. Mais, dans un second temps, ces innovations sont reprises par l'État et les institutions politiques, disposant d'outils législatifs pour entériner les recompositions territoriales face aux enjeux fonciers et limiter les pressions foncières sur les terres agricoles. L'étude de la gouvernance locale, entendue comme la coordination et le partenariat des acteurs locaux (publics et privés) pour résoudre un problème (Mancebo, 2006), permet de mieux comprendre la pérennisation des processus d'innovation dans le domaine de l'agriculture de proximité.

\section{LES PROBLÈMES FONCIERS : QUELLE ÉCHELLE D'INTERVENTION?}

Les collectivités territoriales interviennent avec des logiques différentes dans les pays étudiés.

Dans un pays décentralisé comme la Suède, c’est surtout à l'échelle communale que sont gérés les problèmes fonciers, car l'élu local a le monopole de l'aménagement et de l'occupation des sols sur son territoire. L'outil principal et obligatoire de la politique d'aménagement communal est le plan général (Översiktplan), complété par des plans de détail pour certaines zones. Ces documents déterminent un type d'usage pour chaque zone du territoire. L'autre outil de taille pour les communes est les terres agricoles qu'elles détiennent en propriété, dont la SAU est variable selon les communes. Elles louent alors ces terres agricoles en fermage à des agriculteurs et peuvent influencer l'orientation agricole sur ces exploitations via le contrat de fermage. Enfin, le dernier outil des communes pouvant favoriser le maintien de terres agricoles est constitué des documents environnementaux (Agendas 21 locaux, programmes environnementaux communaux, bilans environnementaux publiés chaque année) qui sont des documents-parapluie pour l'élaboration de tous les documents d'aménagement. Les plans locaux sont fortement influencés par les législations départementales (par exemple pour la régulation des terrains de golfs) mais aussi par les lois nationales qui doivent être respectées lors de la réalisation du plan général communal (loi sur l'environnement, loi sur la gestion des terres agricoles, loi sur les zones naturelles protégées, etc.). Au-delà des lois, l'État suédois a mis en place d'autres outils pour favoriser le développement des surfaces agricoles biologiques et la consommation de produits biologiques : plan de développement de l'agriculture biologique (Riksdagen, 2010), programme d'achats de produits biologiques dans les cantines scolaires et les restaurants administratifs, programme de développement de la gastronomie (Landsbygdsdepartementet, 2010), autant de programmes que les collectivités locales doivent mettre en œuvre. 
La situation est beaucoup plus chaotique en Argentine. Deux problèmes se posent : d'une part, l'activité maraîchère et la préservation des espaces ruraux n'étaient pas, jusqu'alors, la priorité des politiques agricoles, d'autre part, l'aménagement des espaces métropolitains hérite des politiques néolibérales de la décennie 1990, qui avaient favorisé les investissements privés. Promoteurs immobiliers et entreprises ont donc largement investi (et continuent de le faire) les espaces agricoles de la troisième couronne. Or, la majorité des Boliviens ne sont que locataires de leurs terres : les propriétaires peuvent ne pas renouveler leur bail face à une opportunité immobilière. Les municipalités seraient les mieux placées pour arbitrer ces potentiels conflits fonciers et se tenir à un plan d'occupation des sols. Mais les cas diffèrent d'une municipalité à l'autre. Au Sud, une ordonnance municipale limite la construction des complexes d'urbanisation privée dans le but de protéger les espaces productifs maraîchers; au Nord, la gestion semble dépendre de l'intendant en place, qui tantôt encourage la collectivité bolivienne en favorisant l'émergence d'un marché, tantôt continue de signer des permis de construire sur des terres maraîchères. La pression foncière suffit parfois à décider : les prix de location en augmentation constante entraînent le départ des Boliviens et de leurs descendants vers la quatrième couronne.

La comparaison des dynamiques foncières agricoles dans les régions de Buenos Aires et de Stockholm permet de dire que le maintien des espaces maraîchers procède du cas par cas. Les engagements politiques pour préserver les terres agricoles et encourager les nouveaux réseaux d'approvisionnement sont les conditions indispensables de leur pérennité.

\section{LA TERRITORIALISATION DES INNOVATIONS}

Les actions institutionnelles se multiplient en faveur des innovations mais selon des temporalités distinctes.

À Buenos Aires, la prise de conscience qu'il existe un espace périurbain avec ses logiques multiusages spécifiques n'a que trois ans. Le terme même est nouveau dans le vocabulaire institutionnel et académique. En avril 2008, la Présidence de la Nation a octroyé des subventions à l'agriculture périurbaine à destination des producteurs familiaux; début 2009, l'INTA (Institut national de technologie agraire) a créé trois agences périurbaines (Nord, Ouest, Sud) pour superviser une politique périurbaine commune à l'échelle métropolitaine; un projet de parc agricole existe pour la zone Nord de la quatrième couronne périurbaine. Face à la croissance sauvage des années 1990, tout se joue comme si les Boliviens avaient montré leur rôle clé pour l'organisation des périphéries métropolitaines en l'absence de la puissance publique. Aujourd'hui, les autorités politiques ont perçu qu'elles ne pouvaient passer à côté des Boliviens pour la mise en place de politiques périurbaines. Les ateliers, séminaires et formations dispensés par l'INTA rendent compte du nouveau dialogue établi entre les producteurs maraîchers boliviens et les institutions locales pour le maintien d'une agriculture de proximité à Buenos Aires.

Autour de Stockholm, l'étude des politiques d'aménagement de trois communes plus ou moins éloignées de la capitale (Huddinge commune de banlieue sud proche, Södertälje commune de banlieue sud éloignée, et Enköping commune périurbaine à l'Ouest de la capitale) (fig. 2) permet de dire que plus on se rapproche de la capitale, plus les mesures de préservation des terres agricoles et d'encouragement d'une agriculture biologique sont grandes, du fait de la perception directe de la «menace urbaine ». Dans la plupart des cas, l'agriculture est soutenue pour des raisons de protection du patrimoine naturel et des paysages ouverts. Huddinge a eu très tôt une politique ambitieuse de maintien des terres agricoles en utilisant tous les outils dont une commune peut disposer : propriétaire de la totalité de la SAU communale, elle peut orienter son agriculture vers une agriculture biologique selon les objectifs de l'Agenda 21 local et de la politique alimentaire de biologique dans les écoles. À l'inverse, la commune d'Enköping, située à $70 \mathrm{~km}$ de Stockholm, est une commune très agricole avec une SAU importante, mais où la majorité des exploitations pratique une agriculture conventionnelle. La SAU est en propriété privée à $98 \%$; la commune n'a pas d'Agenda 21 local, ni d'objectif de consommation de produits biologiques dans les écoles car 
le poids politique des agriculteurs conventionnels de la commune est tel qu'une mesure favorisant les agriculteurs biologiques serait très mal accueillie. Rien n'est fait pour favoriser le maintien d'une agriculture durable à l'échelle communale. Entre les deux, Södertälje est une commune avec une importante agriculture biodynamique (centre anthroposophique de Järna) mais qui est née d'une initiative privée. La commune n'aide que très faiblement les agriculteurs biologiques à s'installer. Les terres agricoles que la commune possède sont plus considérées comme une réserve foncière pour de futurs projets immobiliers ou pour la construction d'infrastructures de transport, que comme des terres vouées au maintien de l'agriculture. Mais Södertälje s'engage sur la voie de la sensibilisation de ses habitants à l'importance d'une alimentation locale, équitable et biologique. Or l'éloignement par rapport à la capitale correspond aussi à un gradient décroissant de la part de la SAU certifiée biologique dans la SAU communale totale : Huddinge, Södertälje et Enköping (de la commune la plus proche à la plus éloignée de Stockholm) avaient en 2006 une part de SAU biologique par rapport à la SAU totale communale respectivement de $18 \%, 12 \%$ et $11 \%$ (Jordbruksverket, 2009), signe que les politiques locales volontaristes sont bien un facteur important dans le maintien des surfaces agricoles biologiques.

L'investissement personnel des producteurs, des commerçants et, désormais, de la sphère politique change la perspective des réseaux d'approvisionnement de Buenos Aires et Stockholm. Bien plus que des terres agricoles, bien plus que des bâtiments ou des étals en plein air, les espaces maraîchers métropolitains sont des constructions socio-spatiales. Il ne s'agit pas seulement d'un réservoir passif de ressources mais de lieux de création : en ce sens, on peut parler de territoires de l'innovation, contribuant pleinement et durablement à l'alimentation des métropoles.

\section{Conclusion : vers la construction d'une meilleure gouvernance territoriale grâce à l'innovation en réseau?}

L'étude des systèmes maraîchers autour de Stockholm et de Buenos Aires a montré que, dans les deux cas, le maintien d'espaces de production maraîchère, et plus généralement agricole, est le fait de l'organisation des producteurs en réseau, constituant une innovation territoriale majeure. L'agriculture de proximité se maintient malgré la pression foncière métropolitaine, grâce à des transformations profondes (passant notamment par l'intégration de populations marginales) mais aussi à la permanence des réseaux sociaux existants. Les nombreuses interactions entre les agriculteurs et les citadins ainsi que les mobilités personnelles et professionnelles des agriculteurs brouillent les catégories statistiques traditionnelles entre rural et urbain, entre agriculteurs et autres professions : ces dynamiques participent à la recomposition permanente de ces réseaux. Mais les systèmes locaux de gouvernance, et surtout l'articulation entre acteurs privés (agriculteurs, réseaux de commercialisation) et acteurs publics (collectivités territoriales, organismes de recherche), expliquent la plus ou moins grande réussite dans le maintien des espaces agricoles de proximité. Seuls le soutien à et la reconnaissance de ces acteurs privés et de leurs nouvelles formes d'organisation en réseau par les politiques à différentes échelles garantissent le maintien voire le développement du foncier agricole autour des métropoles. Les innovations étudiées dans les systèmes locaux d'approvisionnement urbain en produits maraîchers sont donc porteuses de durabilité pour les territoires à condition que tous les acteurs concernés par l'agriculture de proximité soient impliqués dans la prise de décision et dans l'élaboration des politiques foncières et agricoles, gage d'une bonne gouvernance. Notre éclairage Nord/Sud rappelle que le concept de développement durable appliqué à l'échelle mondiale doit non seulement être évalué à travers ses trois piliers fondamentaux (l'économique, le social, l'environnemental), à valeur égale, mais que le quatrième pilier, celui de la gouvernance, est fondamental dans la réussite de l'application territoriale de ce concept. 


\section{Bibliographie}

Aschan-Leygonie C., 2000. Vers une analyse de la résilience des systèmes spatiaux, L'Espace géographique, vol. $29, \mathrm{n}^{\circ} 1$, p. 64-77.

Benencia R., 2009. Inserción de bolivianos en el mercado de trabajo de la Argentina, Congreso de la Asociación de Estudios Latinoamericanos, Río de Janeiro, 21 p.

Bricas N., Daviron B., 2008. De la hausse des prix au retour du «productionnisme » agricole : les enjeux du sommet sur la sécurité alimentaire de juin 2008 à Rome, Hérodote, n 131, p. 31-39.

Brunet R., Ferras R., Thery H., 1992. Les mots de la géographie. Dictionnaire critique, Montpellier/Paris, GIP RECLUS/La Documentation française, $470 \mathrm{p}$.

Chaleard J.-L., 2005. Marchés et commerce des produits vivriers. Région de Bouaké - Côte d'Ivoire, Paris, coll. « Mémoires et Documents de l'UMR PRODIG », 92 p.

Douzant-Rosenfeld D., Grandjean P. (dir.), 1996. Nourrir les métropoles d’Amérique latine, Paris, L’Harmattan, $302 \mathrm{p}$.

FAO, 2009. Aliments pour les villes, Rome, 8 p.

Fleury A., Donadieu P., 1997. De l'agriculture périurbaine à l'agriculture urbaine, Le courrier de l'environnement de l'INRA, n 31, p 45-61.

Кеват C., 2008. Transformaciones en la horticultura platense. Una mirada a través de los censos, Realidad económica, nº 237, p. 110-134.

Guiv C., 2007. Pratiques folkloriques dans les « Terres de l'Ebre » : représentations et mises en scène de la ruralité, Norois, no 204/3, p. 39-52.

Hochedez C., 2008. Le bonheur est dans le panier, Géocarrefour, vol. 83, nº 3, p. 225-233.

Houtard F. (dir.), 2009. Etat des résistances dans Le Sud-2009. Face à la crise alimentaire. Alternatives Sud, $n^{\circ}$ 15/4, Paris, Editions Syllepse, 226 p.

Holling C. S., 1973. Resilience and stability of ecological systems, Annual review of Ecology ans Systematics, $\mathrm{n}^{\circ} 4$, p. 1-23.

JordBruksDePARTEMENTET, 2006. Förslag till Landsbygdsprogram för Sverige, år 2007-2013, Stockholm, Jordbruksdepartementet, $232 \mathrm{p}$.

Jordbruksverket, 2009. Jordbruksstatistisk årsbok 2009, Örebro, Statistiska centralbyrån, 389 p.

LANDSBygdSDEPARTEMENTET, 2010. Sverige - det nya matlandet. Uppdaterad handlingsplan. Nya jobb genom god mat och upplevelser, Stockholm, Regeringskansliet, 16 p.

Le GaLl J., 2007. Quand les migrants construisent des territoires: le marché de gros de la Matanza, nouveau territoire bolivien de Buenos Aires, Second Congrès bisannuel du GIS Amérique latine, Rennes.

Le Gall J., García M., Barsky A., 2009. Trayectorias bolivianas, redes hortícolas. El papel de los migrantes en la recomposición territorial de los espacios rurales del AMBA, Seminario internacional « Nuevas trayectorias de las migraciones internacionales en América latina y en Europa »(Conicet/DIGEO), Buenos Aires, 17 novembre 2009.

Mancebo F., 2006. Le développement durable, Armand Colin, Paris, collection « U », 269 p.

Ministerio de Asuntos agrarios, ministeria de Economia, Gobierno de la provincia de Buenos Aires, 2006. Censo Hortiflorícola de la Provincia de Buenos Aires 2005, La Plata, Dirección Provincial de Estadística, 115 p.

Pierre G., Madeline Ph., Margetic C., Croix N., Bermond M., Peltier C., 2008. Durabilité, agricultures et territoires : quels questionnements pour les ruralistes d'universités de l'Ouest?, Géocarrefour, vol. 83, $n^{\circ} 3$, p. 245-250.

Pizarro C., 2009. Olor a negro. Discurso, discriminación y segmentación étnica en el lugar de trabajo, Communication lors de IV Coloquio de investigadores en estudios del discurso, Córdoba.

Poulot Monique, 2008. Les territoires périurbains : « fin de partie » pour la géographie rurale ou nouvelles perspectives?, Géocarrefour, vol. 83/4, p. 269-278.

RiKsDagen, 2010. Uppföljning av ekologisk produktion och offentlig konsumtion, 2010/1 1, RFR1, Stockholm, Riksdagstryckeriet, 149 p. 